\title{
MOLECULAR HYDROGEN EMISSION IN EMBEDDED FLOWS
}

\author{
JOCHEN EISLÖFFEL \\ Institute for Astronomy, University of Hawaii, \\ 2680 Woodlawn Drive, Honolulu, HI 96822, U.S.A. \\ Max-Planck-Institut für Astronomie, \\ Königstuhl 17, D-69117 Heidelberg, Germany \\ Thüringer Landessternwarte Tautenburg, \\ Sternwarte 5, D-07778 Tautenburg, Germany
}

\begin{abstract}
This overview of recent observations of molecular hydrogen $\left(\mathrm{H}_{2}\right)$ in outflows from young stars discusses the morphology, the excitation and the kinematics of the $\mathrm{H}_{2}$ gas in these flows. A comparison between the $\mathrm{H}_{2}$ and $\mathrm{CO}$ outflows will lead us to the conclusion that highly-collimated jets may drive the latter. We also discuss the mechanisms which can entrain ambient molecular gas into the jet flow.
\end{abstract}

\section{Introduction}

Bipolar outflows from young stars are among the most spectacular manifestations of star formation. We now recognize that they are one of the fundamental astrophysical processes that regulate star formation both in single objects and on scales of molecular clouds. Outflows are observed in the emission lines of many atoms and molecules in various excitational states. It is widely believed that we see radiatively cooling gas, which has been excited by shocks in the supersonic flows or by their interaction with the ambient medium.

In regions of relatively low extinction optical emission, e.g. in the $\mathrm{H} \alpha$ and [SII] $\lambda \lambda 6716,6731$ lines, typical of Herbig-Haro objects, can be seen. More deeply embedded flows are more accessible in tracers at longer wavelengths: molecular hydrogen $\left(\mathrm{H}_{2}\right)$, the most abundant molecule in the Universe, is an important tracer of low-velocity shocks at near- to mid-infrared wave- 


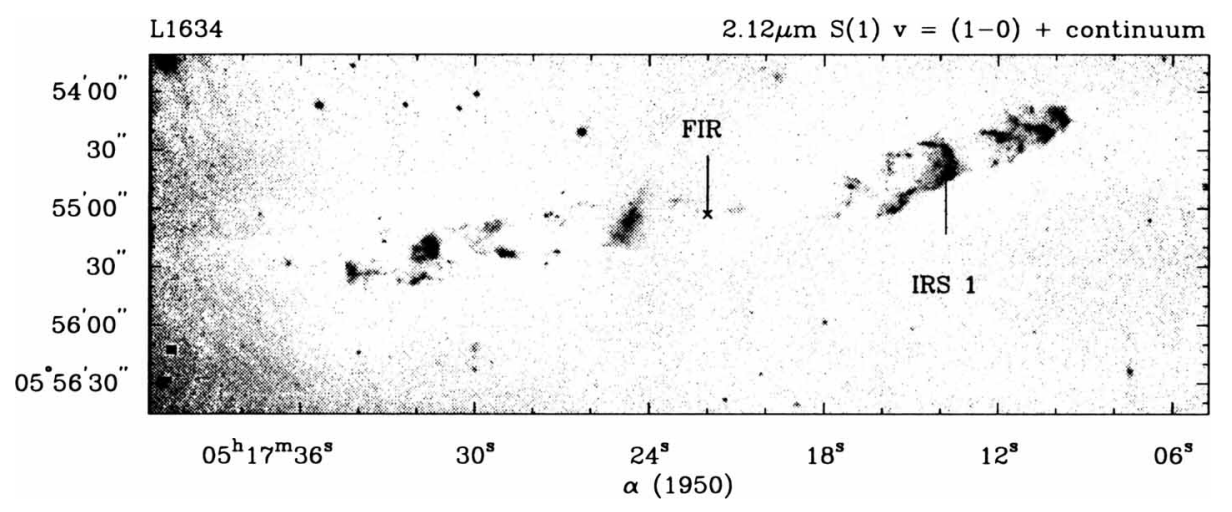

Figure 1. The L1634 outflow in the 1-0 S(1) line of $\mathrm{H}_{2}$ at $2.12 \mu \mathrm{m}$ (from Hodapp \& Ladd 1995).

lengths. Because of its short cooling time (of the order of a year) it illustrates regions where interactions are currently taking place: the $\mathrm{H}_{2}$ emission is like the sparks that fly when outflowing gas hits something in its way. On the other hand, $\mathrm{CO}$, which is excited in cool gas $\left(\mathrm{T}_{e x} \geq 5 \mathrm{~K}\right)$, shows us the coadded history of the interactions at submm- and mm-wavelengths. All of this information is vital if we are to understand how winds from young stars interact with the surrounding molecular gas, how the wind energy and momentum is dissipated, if and how ambient gas is entrained, and consequently if and how winds can drive massive molecular outflows.

Here I will give an overview of the morphology, excitation, and kinematics of $\mathrm{H}_{2}$ in embedded outflows. Then, I will compare $\mathrm{H}_{2}$ and $\mathrm{CO}$ in outflows, and finally I will describe some recent results on entrainment of ambient gas into the flows.

\section{Morphology of $\mathbf{H}_{2}$ in outflows}

Molecular hydrogen has been observed in optically visible Herbig-Haro (HH) objects both by direct imaging (see, e.g. Hartigan, Curiel \& Raymond 1989; Stapelfeldt et al. 1991; Eislöffel et al. 1994; Davis, Mundt \& Eislöffel 1994; Noriega-Crespo et al. 1996) and by spectroscopy (see, e.g., Gredel 1994, 1996; Fernandes \& Brand 1995). However, not all HH objects are bright in $\mathrm{H}_{2}$ emission, most likely because many are already rather evolved and have broken out of their molecular cloud cores. Deeply embedded outflows from Class 0 or Class I sources are probably much younger and are still 
ploughing their way through a denser molecular environment (see Fig. 1). Such flows usually show one or several bright and bow-shaped knots in $\mathrm{H}_{2}$ (see, e.g., Bally, Lada \& Lane 1993; Hodapp 1994; Davis \& Smith 1996a; Eislöffel et al. 1996; Ladd \& Hodapp 1997). These bright knots are thought to be the working surfaces of the flows. In addition, the flows often show faint and more diffuse $\mathrm{H}_{2}$ emission stretching from the bows back towards the source along both sides of the flow axis (see, e.g., Davis \& Eislöffel 1995; Dent, Matthews \& Walter 1995; Hodapp \& Ladd 1995). Such condensations may result from interaction of the flow with the ambient medium in a shear layer along the flow channel walls.

\section{Excitation of $\mathbf{H}_{2}$ in outflows}

Various types of shock structures may be present in the shock waves that form in the flows or in their violent interaction with the ambient medium. If the shock speed is sufficiently high a "J-shock" may arise, in which a jump in velocity, density, temperature, pressure and entropy takes place, so that the shock front appears as a discontinuity. On the contrary a "C-shock", in which the shock energy is dissipated via ambipolar diffusion between ions and neutrals, will show continous changes of quantities within the shock structure (e.g. Draine \& McKee 1993). To understand the shock structures better, a detailed knowledge of the excitation state of the gas, as measured by the column density of $\mathrm{H}_{2}$ in each energy level, is clearly necessary.

Several outflows have been studied in detail so far, with differing results: While observations of OMC-1 peak 1 (Nadeau \& Geballe 1979; Moorhouse et al. 1990) could be well fitted by C-shock models (Smith, Brand \& Moorhouse 1991; Smith 1991), J-shocks seem to be present in the bow shock of HH 91 (Smith 1994). Observations of HH 1 by Noriega-Crespo \& Garnavich (1994) were consistent with either J-type or C-type shocks, while Gredel (1994, 1996) could fit his data on various outflows with models of slabs of gas at a single temperature. On the other hand, Fernandes \& Brand (1995) required a combination of C-type bow shocks and fluorescence to explain the excitation in $\mathrm{HH} 7$. If we assume that similar processes are at work in all objects, these confusing results may follow from the fact that all the spectroscopic observations covered only a limited range in upper level energy of $\mathrm{H}_{2}$, or that they lacked spatial resolution (or both). Recently, Eislöffel et al. (1996) tried to overcome these limitations in a different observational approach: the Cep E outflow was imaged at high $\mathrm{S} / \mathrm{N}$ with narrow-band filters in the three near-infrared $\mathrm{H}_{2}$ lines 1-0 S(1), 2-1 S(1), and 3-2 $\mathrm{S}(3)$ (and in the nearby continuum), lines which cover a wide range in upper level energy $(6950 \mathrm{~K}$ to $19080 \mathrm{~K})$. These lines were found to show relative brightnesses close to $1: 0.1: 0.01$, the line ratios being constant to within $1 \%$ everywhere. 

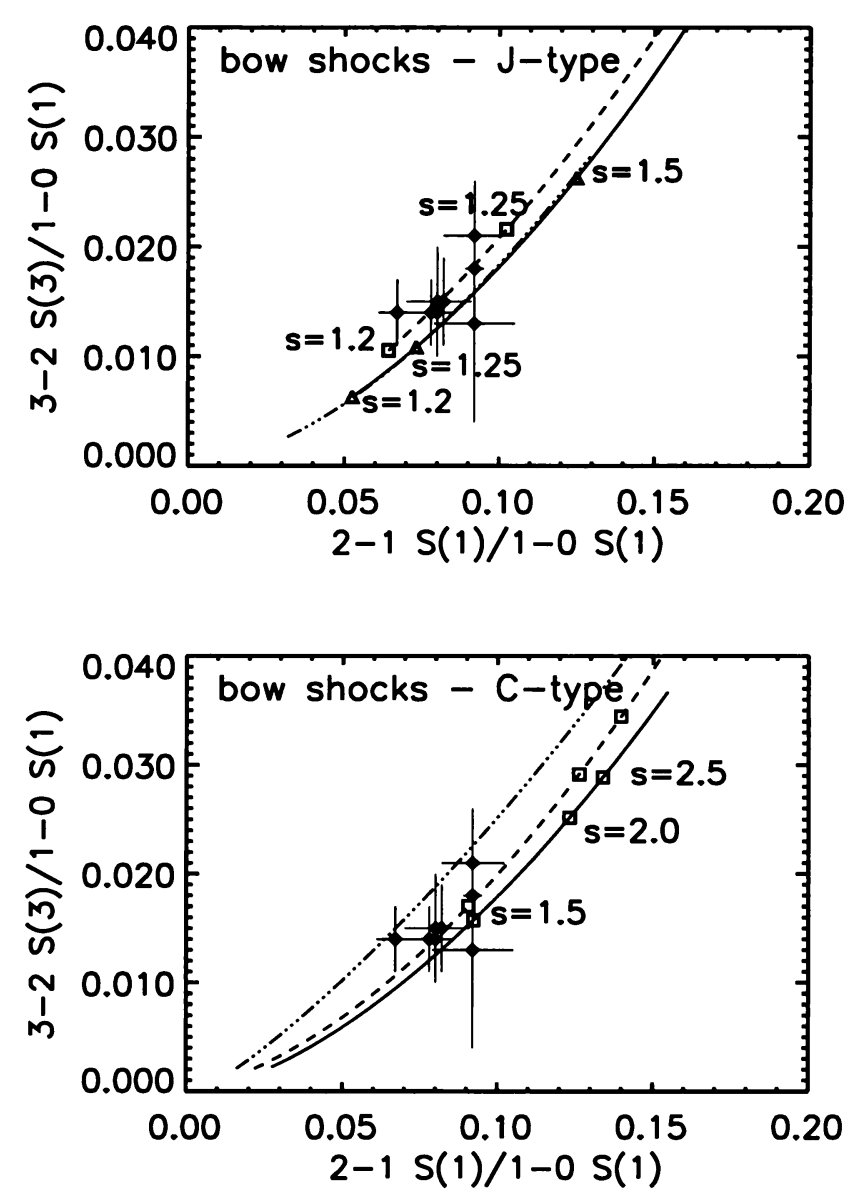

Figure 2. upper panel: J-type bow shock models superimposed on Cep E line ratios. lower panel: C-type bow shock models superimposed on the same data (from Eislöffel et al. 1996).

Comparison of the data with models of slabs of gas at one or two fixed temperatures cannot account for the observed line ratios, nor can fluorescence nor planar J- or C-type shocks. J-type bow shocks (Fig. 2, upper panel) cannot be excluded althought most data points fall above the theoretical curve, and the model bows are very wide with a shape parameter $\mathrm{s}=1.2$ - 1.3 (where the bow shape is defined as $z=r^{s}$ ). Numerical simulations by Suttner et al. (1996) produce shape parameters of $\mathrm{s}=1.5-1.7$. C-type bow shocks seem to be consistent with the observations of a large range of densities (Fig. 2 lower panel). They require a shape parameter of about $\mathrm{s}$ 


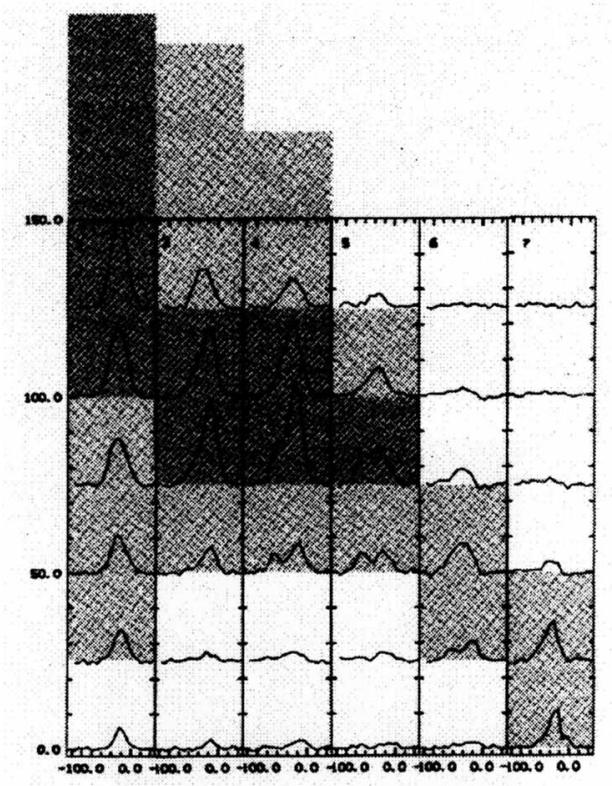

Figure 3. $\mathrm{H}_{2}$ 1-0 S(1) spectra from regions around knot A in L1448. The spectra are superimposed onto a grey-scale image of the bow shock A. Double-peaked line profiles, typical of bow shocks, are clearly indicated (from Davis \& Smith 1996a).

$=1.5$, in agreement with the numerical simulations. However, the rather constant line ratios observed across the entire outflow region are not easily understood. These would not be expected for a resolved bow shock. It has therefore been suggested that the large bows in Cep E may have broken up due to instabilities, and may now consist of unresolved mini-bow shocks which move at more or less the same speed.

Various accepted programmes for ISO will do imaging and spectroscopy of purely rotationally excited lines of $\mathrm{H}_{2}$ with upper level energies from $1680 \mathrm{~K}$ to $4600 \mathrm{~K}$ in the thermal infrared. This will extend the total observed range of upper level energy to more than a factor of 10 and will constrain the excitation models further than is possible at present.

\section{Kinematics of $\mathbf{H}_{2}$ in outflows}

The kinematics of the $\mathrm{H}_{2}$ gas studied with high-resolution spectroscopy provides another invaluable means to understand the shock structures: wide, double-peaked profiles are expected in bow shocks, whereas wide, but single-peaked Gaussian profiles are expected in turbulent boundary layers, and narrower, symmetric lines are expected in planar shocks (Smith \& 

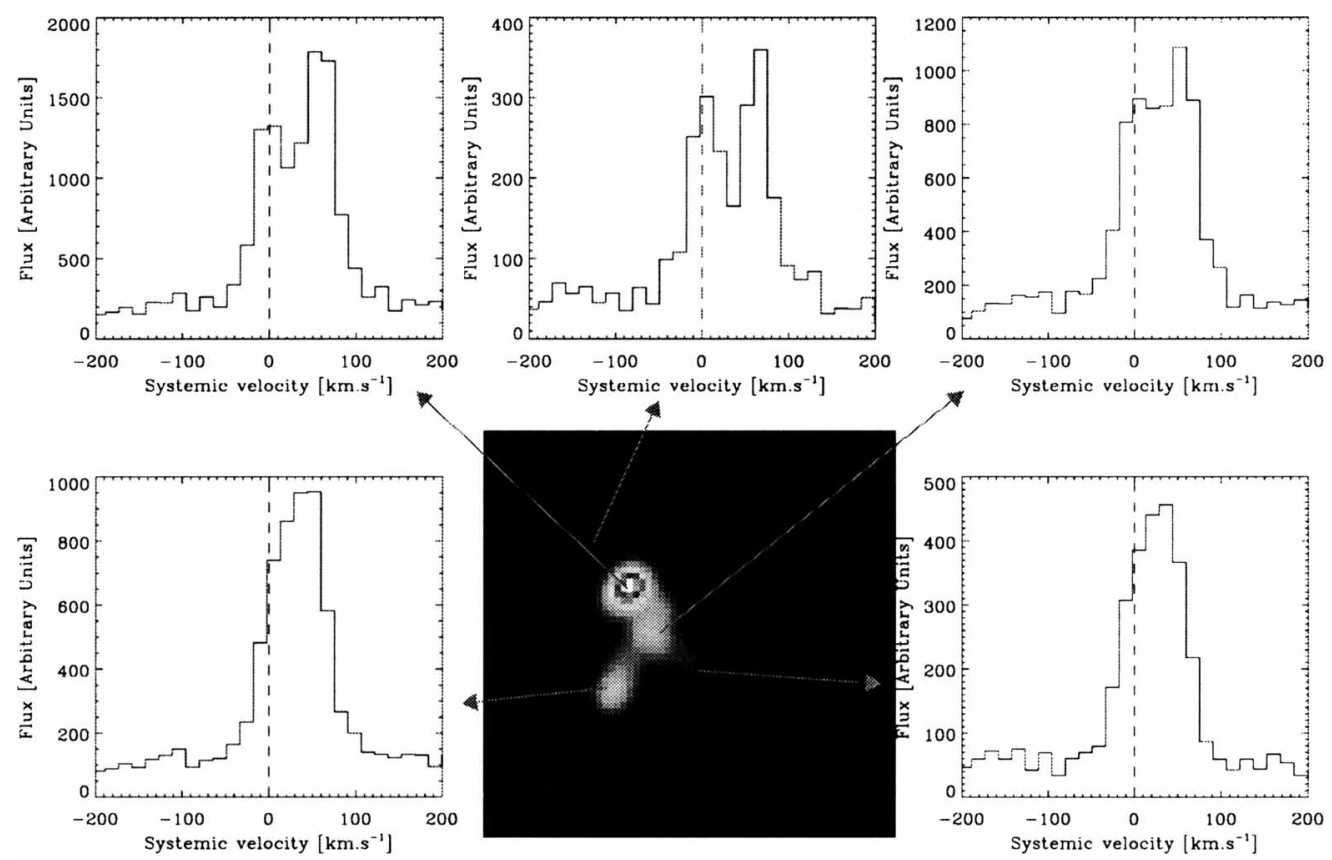

Cep:- North

Figure 4. Spectro-image of the northern bow $\mathrm{N} 1$ in Cep E in the $\mathrm{H}_{2}$ 1-0 S(1) line, taken with BEAR at CFHT. Several of the line profiles extracted at various positions are double-peaked, typical of bow shocks (Eislöffel et al., in prep.).

Brand 1990a, 1990b). Centroid velocities and observed asymmetries in the line profiles may be used to predict shock speeds and outflow orientations (see e.g. Hartmann \& Raymond 1984). This second parameter is of vital importance to outflow studies at all wavelengths (optical through to radio), since outflow energetics may only be estimated (from radial velocities) if one knows the inclination angle of the flow.

So far, only a few outflows have been studied in the $\mathrm{H}_{2}$ 1-0 S(1) line at high spectral resolution, thus resolving the line profiles. They include HH 1/2 and HH 7-11 (Zinnecker et al. 1989), L1448 (see Fig. 3, and Davis \& Smith 1996a), DR21 (Davis \& Smith 1996b), HH32 (Davis, Eislöffel \& Smith 1996) and Cep E (see Fig. 4, Eislöffel et al., in prep.).

Evidence for turbulent shear layers between the flow and its environment is seen in the spectra of DR21 and parts of L1448, which show Gaussian line profiles. On the other hand, the bright condensations in L1448 and Cep E show wide, double-peaked profiles, typical of bow shocks. Also the profiles in HH 32 are consistent with a high-velocity bow shock pointed away from 


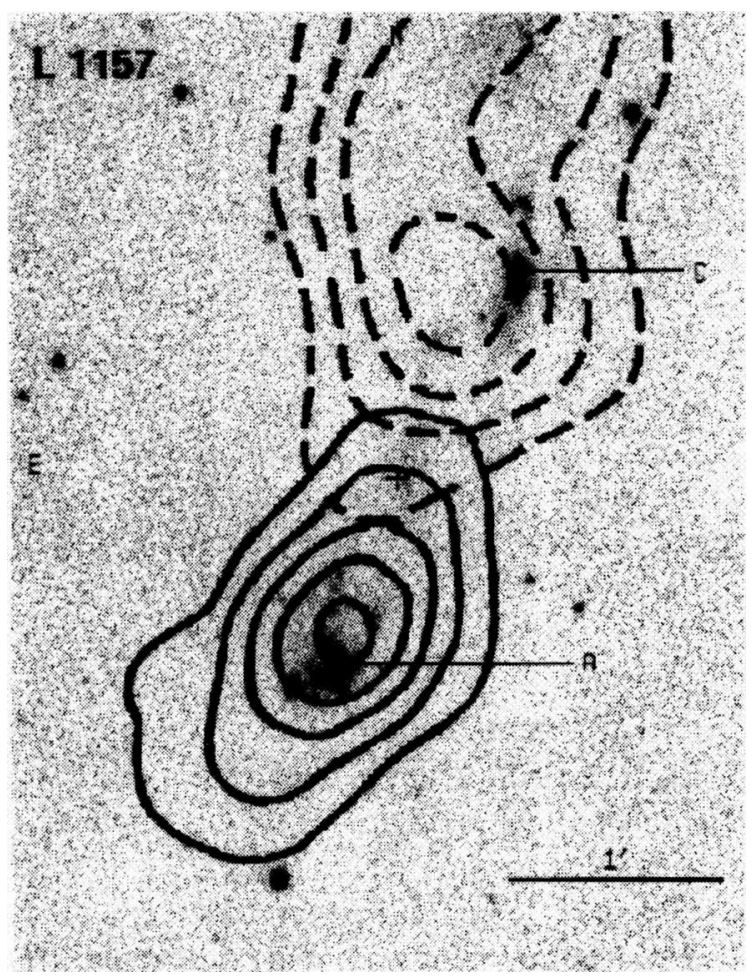

Figure 5. $\mathrm{H}_{2}$ 1-0 S(1) image of the L1157 outflow region with contours of the CO 1-0 outflow. The $\mathrm{H}_{2}$ regions $\mathrm{A}$ and $\mathrm{C}$ are clearly associated with the peaks in the $\mathrm{CO}$ outflow lobes (from Davis \& Eislöffel 1995).

us.

These observations bring up the question whether the $\mathrm{H}_{2}$ emission in these outflows derives from internal or "working-surface" bow shocks, or whether supersonic turbulence in a "mixing layer" around the flows plays a dominant role. A comparison of the $\mathrm{H}_{2}$ and $\mathrm{CO}$ in the outflows may help to answer this question.

\section{Comparison of $\mathrm{H}_{2}$ and $\mathrm{CO}$ outflows}

Recent observations have shown that at least some CO outflows are quite well-collimated (see the review by Bachiller 1996 and references therein). When overlaying $\mathrm{CO}$ maps onto $\mathrm{H}_{2}$ images it becomes evident that $\mathrm{CO}$ and $\mathrm{H}_{2}$ gas are intimately related to each other (see Fig. 5 and other examples in 

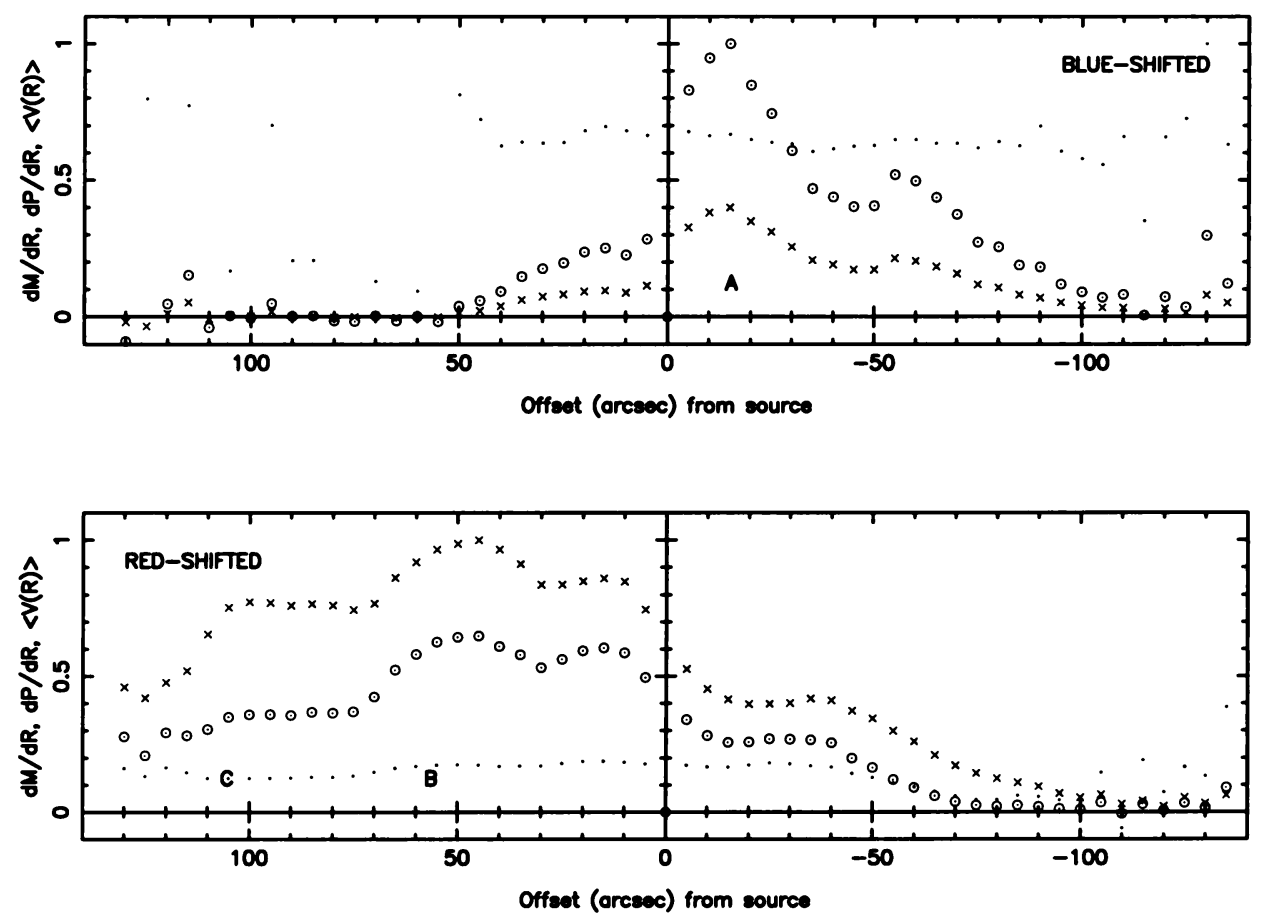

Figure 6. Distribution of mass (integral $\mathrm{T}_{b} \cdot \mathrm{dv}$ - crosses), momentum (integral $\mathrm{T}_{b} . \mathrm{v} . \mathrm{dv}$ - dots+circles), and mean velocity (dots) along theRNO 15-FIR outflow axis. Profiles for the blue-shifted $(\mathrm{v}<2.0 \mathrm{~km} / \mathrm{s})$ and red-shifted $(\mathrm{v}>7.4 \mathrm{~km} / \mathrm{s})$ high-velocity gas are plotted, measured at 5 " intervals, from the sum of spectra in strips perpendicular to, though centred on, the outflow axis. $\mathrm{dM} / \mathrm{dR}, \mathrm{dP} / \mathrm{dR}$ and $\langle\mathrm{V}(\mathrm{R})>$ are normalised to the maximum measured values. The offsets are in arcseconds from RNO 15-FIR (positive ' $x$ ' is towards the northeast). The positions of the molecular shocks along the outflow axis are labelled A, B, and C.

Davis \& Eislöffel 1995). In almost all outflows the CO peaks just upstream of the bright $\mathrm{H}_{2}$ bows. The other $\mathrm{H}_{2}$ condensations which are seen between the bright $\mathrm{H}_{2}$ bows and the outflow source turn out to line up along the sides of the $\mathrm{CO}$ outflow lobes. These observations suggest that the $\mathrm{CO}$ outflows consist of ambient gas that has been entrained by a highly-collimated "jet".

\section{Entrainment mechanisms}

How can ambient molecular gas be entrained by collimated jets to form massive, molecular (CO) outflows? Theoretical studies suggest that ambient gas may be accelerated, either in a turbulent mixing layer along the length of the jet or a jet segment (Cantó \& Raga 1991; Masson \& Chernin 1992), 
or through the bow shock at the head of the jet (Masson \& Chernin 1993; Raga \& Cabrit 1993). In the turbulent model, a shear layer develops along the interface between the supersonic jet and its surroundings which thickens with distance from the source, as jet material and ambient gas enter this layer. Conversely, entrainment in the bow shock or "prompt" model is quite localised. Here, the ambient, molecular gas swept up through each bow shock cools rapidly, and although some of the gas may spill down the sides of the jet, a high-velocity "clump" will develop just behind each shock front.

In the turbulent entrainment model, the momentum per unit length should increase with distance from the source, as more of the jet momentum is transfered to the swept up molecular gas (Chernin \& Masson 1995). In the prompt entrainment model, however, the momentum should decrease with distance from the source if the flow "accelerates" out of a dense protostellar core into lower density gas.

Recent CO data taken by Davis et al. (1997a) allow them to distinguish between entrainment models in the RNO15-FIR outflow. This seemingly rather simple outflow consists of a sequence of compact line emission features, which imply the presence of a highly-collimated, bipolar outflow driven by RNO15-FIR (Davis et al. 1997b). The spatial coincidence between three peaks in the $\mathrm{CO}$ map and three corresponding $\mathrm{H}_{2}$ shocks suggests that (a) these $\mathrm{H}_{2}$ shocks are those which entrain much of the ambient gas to form the $\mathrm{CO}$ outflow, and that (b) the prompt entrainment mechanism dominates over turbulent entrainment. Fig. 6 shows the mass per unit length $(\mathrm{dM} / \mathrm{dR})$, momentum per unit length $(\mathrm{dP} / \mathrm{dR})$, and mean velocity $(<\mathrm{V}(\mathrm{R})\rangle=[\mathrm{dP} / \mathrm{dR}] /[\mathrm{dM} / \mathrm{dR}])$ along the outflow axis, integrated across the width of the flow and over the high-velocity blue-shifted and red-shifted line wings. In both the northeastern, red-shifted flow lobe, and the southwestern, blue-shifted flow lobe, the mass and momentum decrease with distance from the source. This decrease is most dramatic in the blueshifted lobe (at negative offsets in Fig. 6). Also, the molecular $\left(\mathrm{H}_{2}\right)$ shocks observed along the flow lobe coincide with peaks in these distributions. These $\mathrm{H}_{2}$ features therefore probably represent the entraining bow shocks. Indeed, it seems very likely that prompt or "bow shock" entrainment dominates in this particular outflow.

\section{Conclusions}

In this overview of molecular hydrogen in outflows from young stars we have seen that the $\mathrm{H}_{2}$ is observed as bright bow shocks at the ends of the flows (or flow segments) and as fainter emission along the flow axis. The excitation mechanism of this $\mathrm{H}_{2}$ gas is not yet clear. A comparison of the $\mathrm{H}_{2}$ and $\mathrm{CO}$ maps shows that the $\mathrm{CO}$ is probably entrained by a highly-collimated jet. 
High-resolution spectroscopic studies of the kinematics of both $\mathrm{H}_{2}$ and $\mathrm{CO}$ indicate that this happens mostly via prompt entrainment in the leading bows, and to a minor extent also via turbulent entrainment in shear layers along the flow axis.

Acknowledgements: I would like to thank Chris Davis very much for our pleasant collaboration on molecular hydrogen and embedded outflows. My thanks also go to Mike Smith, Jérôme Bouvier, Reinhard Mundt, Tom Ray and François Rigaut for their help and participation in many projects. Finally, I wish to thank the organizers for their support.

\section{References}

Bachiller, R. 1996, Ann. Rev. Astron. Astrophys. 34, 111

Bally, J., Lada, E.A., Lane, A.P. 1993, ApJ 418, 322

Cantó, J., Raga, A.C. 1991, ApJ 372, 646

Chernin, L.M., Masson C.R. 1995, ApJ 455, 182

Davis, C.J., Eislöffel, J. 1995, A\&A 300, 851

Davis, C.J., Eislöffel, J., Ray, T.P., Jenness, T. 1997a, A\&A, in press

Davis, C.J., Eislöffel, J., Smith, M.D. 1996, ApJ 463, 246

Davis, C.J., Mundt, R., Eislöffel, J. 1994, ApJ 437, L55

Davis, C.J., Smith, M.D. 1996a, A\&A 309, 929

Davis, C.J., Smith, M.D. 1996b, A\&A 310, 961

Davis, C.J., Ray, T.P., Eislöffel, J., Corcoran, D. 1997b, A\&A, in press

Dent, W.R.F., Matthews, H.E., Walther, D.M. 1995, MNRAS 277, 193

Draine, B.T., McKee, C.F. 1993, Ann. Rev. Astron. Astrophys. 31, 373)

Eislöffel, J., Davis, C.J., Ray, T.P., Mundt, R. 1994, ApJ 422, L91

Eislöffel, J., Smith, M.D., Davis, C.J., Ray, T.P. 1996, AJ 112, 2087

Fernandes, A.J.L, Brand, P.W.J.L. 1995, MNRAS 274, 639

Gredel, R. 1994, A\&A 292, 580

Gredel, R. 1996, A\&A 305, 582

Hartigan, P., Curiel, S., Raymond, J. 1989, ApJ 347, L31

Hartmann, L.W., Raymond, J. 1984, ApJ 276, 560

Hodapp, K.-W. 1994, ApJS 94, 615

Hodapp, K.-W., Ladd, E.F. 1995, ApJ 453, 715

Ladd, E.F., Hodapp, K.-W. 1997, ApJ 474, 749

Masson C.R., Chernin, L.M. 1992, ApJ 387, L47

Masson C.R., Chernin, L.M. 1993, ApJ 414, 230

Moorhouse, A., Brand, P.W.J.L., Geballe, T.R., Burton, M.G. 1990, MNRAS 242, 88

Nadeau, D., Geballe, T.R. 1979, ApJ 230, L169

Noriega-Crespo, A., Garnavich, P.M. 1994, Rev. Mex. AA 28, 173

Noriega-Crespo, A., Garnavich, P.M., Raga, A.C., Cantó, J., Böhm, K.-H. 1996, ApJ 462,804

Raga, A.C., Cabrit, S. 1993, A\&A 278, 267

Smith, M.D. 1991, MNRAS 253, 175

Smith, M.D. 1994, A\&A 289, 256

Smith, M.D., Brand, P.W.J.L. 1990a, MNRAS 243, 498

Smith, M.D., Brand, P.W.J.L. 1990b, MNRAS 245, 108

Smith, M.D., Brand, P.W.J.L., Moorhouse, A. 1991, MNRAS 248, 730

Stapelfeldt, K.R., Scoville, N.Z., Beichman, C.A., Hester, J.J., Gautier, T.N. 1991, ApJ 371,226

Suttner, G., Smith, M.D., Yorke, H.W., Zinnecker, H. 1996, A\&A 318, 595

Zinnecker, H., Mundt, R., Geballe, T.R., Zealey, W.J. 1989, ApJ 342, 337 